\title{
Papel do enfermeiro na assistência ao paciente com esquizofrenia
}

\author{
Role of the nurses in care for patients with schizophrenia \\ Papel de las enfermeras en la atención del paciente con esquizofrenia
}

Bruna Eduarda Alves da Silva

ORCID: https://orcid.org/0000-0002-0059-1475

Universidade Ceuma, Brasil

E-mail: bruninhaseledor@gmail.com

Suzanny Oliveira Silva

ORCID: https://orcid.org/0000-0002-2942-8401

Universidade Ceuma, Brasil

E-mail: enfsuzanny@hotmail.com

Gabriela Caroline Silva Queiroz

ORCID: https://orcid.org/0000-0003-4115-9176

Universidade Ceuma, Brasil

E-mail: gabqrzz@gmail.com

Antônia Eliene Alves Guimarães

ORCID: https://orcid.org/0000-0002-7699-9151

Universidade Ceuma, Brasil

E-mail: elienetcenfermagem@gmail.com

Keiliane Pinheiro Silva Barros

ORCID: https://orcid.org/0000-0002-0477-9766 Faculdade Pitágoras, Brasil

E-mail: keilianepinheiromk@gmail.com

Antônio Silva Machado

ORCID: https://orcid.org/0000-0002-5403-7672

Faculdade de Imperatriz, Brasil

E-mail: neto.maxado@gmail.com

Artur de Souza Veras

ORCID: https://orcid.org/0000-0002-6160-8736 Universidade do Estado do Pará, Brasil E-mail: lasferro1@gmail.com

Patrícia dos Santos Silva Queiroz

ORCID: https://orcid.org/0000-0002-9587-1786 Unidade de Ensino Superior do Sul do Maranhão, Brasil E-mail: patriciasqueiroz@gmail.com

\begin{abstract}
Resumo
Atualmente com a Reforma Psiquiátrica existem maiores e melhores dispositivos para se trabalhar com pacientes portadores de transtornos mentais e a enfermagem desenvolve papel significativo quanto aos cuidados oferecidos a esse usuário. A esquizofrenia é uma doença de cunho mental que é definida por distorções do pensamento, percepção e afetos. Com isso, o objetivo deste trabalho é relatar a importância do cuidado de enfermagem frente ao paciente esquizofrênico, através de um estudo bibliográfico de caráter descritivo. Os critérios de inclusão foram, artigos, manuais do Ministério da Saúde, monografias, dissertações, teses disponíveis na íntegra, publicados e escritos na língua portuguesa, coerentes com o tema da pesquisa e publicados no período de 2015 à 2020. Os critérios de exclusão foram: materiais que não abordavam a temática proposta e/ou não atendiam aos critérios de inclusão descritos anteriormente e fora do período estipulado para a pesquisa. A enfermagem é essencial frente a esses casos pois é este profissional que traça diagnósticos de enfermagem que elaboram a sistematização visando fatores de risco e possíveis agravos. Através desta pesquisa foi possível conhecer sobre a evolução dos tratamentos psiquiátricos e um pouco mais sobre transtornos esquizofrênicos. Sobretudo, este trabalho possibilitou o alcance de uma visão holística sobre a atuação de enfermagem frente aos cuidados com esses pacientes, não apenas com enfoque técnico-medicamentoso.
\end{abstract}

Palavras-chave: Enfermagem; Saúde mental; Esquizofrenia.

\begin{abstract}
Currently, with the Psychiatric Reform, there are bigger and better devices to work with patients with mental disorders and nursing plays a significant role in the care offered to this user. Schizophrenia is a mental illness that is defined by distortions of thought, perception and affect. Thus, the objective of this work is to report the importance of nursing care for schizophrenic patients, through a descriptive bibliographic study. Inclusion criteria were articles, manuals of the Ministry of Health, monographs, dissertations, theses available in full, published and written in Portuguese, consistent
\end{abstract}


with the research theme and published in the period from 2015 to 2020 . The exclusion criteria were : materials that did not address the proposed theme and/or did not meet the inclusion criteria described above and outside the period stipulated for the research. Nursing is essential in these cases because it is this professional who draws nursing diagnoses that elaborate the systematization aiming at risk factors and possible injuries. Through this research it was possible to know about the evolution of psychiatric treatments and a little more about schizophrenic disorders. Above all, this work made it possible to reach a holistic view of the nursing role in caring for these patients, not just with a technicalmedicinal focus.

Keywords: Nursing; Mental health; Schizophrenia.

\section{Resumen}

Actualmente, con la Reforma Psiquiátrica, existen más y mejores dispositivos para trabajar con pacientes con trastornos mentales y la enfermería juega un papel importante en la atención que se brinda a este usuario. La esquizofrenia es una enfermedad mental que se define por distorsiones del pensamiento, la percepción y el afecto. Así, el objetivo de este trabajo es dar a conocer la importancia del cuidado de enfermería al paciente esquizofrénico, a través de un estudio bibliográfico descriptivo. Los criterios de inclusión fueron artículos, manuales del Ministerio de Salud, monografías, disertaciones, tesis disponibles íntegramente, publicadas y redactadas en portugués, acordes con el tema de investigación y publicadas en el período de 2015 a 2020. Los criterios de exclusión fueron: materiales que no no abordó el tema propuesto y / o no cumplió con los criterios de inclusión descritos anteriormente y fuera del período estipulado para la investigación. La enfermería es fundamental en estos casos porque es este profesional quien elabora los diagnósticos de enfermería que elaboran la sistematización apuntando a los factores de riesgo y posibles lesiones. A través de esta investigación se pudo conocer sobre la evolución de los tratamientos psiquiátricos y un poco más sobre los trastornos esquizofrénicos. Sobre todo, este trabajo permitió alcanzar una visión holística del rol de la enfermería en el cuidado de estos pacientes, no solo con un enfoque técnico-médico.

Palabras clave: Enfermería; Salud mental; Esquizofrenia.

\section{Introdução}

Com relação a estruturação da atenção psicossocial, Amarante e Nunes (2018) destacam que a Reforma Psiquiátrica Brasileira (RPB) se afigura como um processo social abrangente, cujos objetivos centrais ainda não efetivados por completo são a formação de um novo lugar social para os portadores de transtornos mentais e a transformação das práticas oficializadas às quais os mesmos, maiores números, eram submetidos sob a ajuda do modelo hospitalocêntrico, predominante no país até o início dos anos 2000.

Desta maneira, a operacionalização da RPB, desde o cenário técnico-assistencial, depende da organização de uma rede formada por variados pontos de atenção, hoje em dia denominada Rede de Atenção Psicossocial - RAPS. Corresponde que serviços públicos de saúde relativos à Atenção Primária em Saúde (APS) - como as UBSs - também devem incluir tal rede, afim que esta possa prestar um cuidado territorial conciliável com a globalidade das demandas dos usuários e valorizá-los como cidadãos. (Brasil, 2014).

A assistência a pessoas em adoecimento mental é uma das atribuições dos serviços de saúde pública, sejam eles especializados em saúde mental (Centros de Atenção Psicossocial (CAPSs)) ou não (Unidades Básicas de Saúde (UBSs). (Rosa., et al).

Uma das indicações quanto a reabilitação psicossocial é que os locais de atendimentos possam possibilitar a criação do cotidiano a partir do diálogo, envolvimento de profissionais, familiares e usuários propondo a educação da sociedade. (Amorim \& Otani, 2015; Hirdes; Kantorski apud Macedo et al., 2018).

$\mathrm{O}$ enfermeiro e sua equipe têm o dever de dirigir suas ações para atender as necessidades apresentadas pelo esquizofrênico. Na prática, verifica-se que enfermeiros psiquiátricos, frequentemente, encontram dificuldades em realizar os cuidados de enfermagem aos esquizofrênicos, de acordo com as características dessa doença mental. (Castro; Furegato, 2010 apud Santos, 2017).

A esquizofrenia é caracterizada por um adoecimento mental grave. Os sintomas incluem transtornos cognitivos, emocionais e comportamentais que modificam a percepção, raciocínio, comunicação, afeto, atenção, entre outras manifestações. 
A gravidade da doença diz respeito, a situação em que o doente dificilmente consegue voltar ao seu estado que antecede a doença. American Psychiatric Association, 2013; Silva, 2006 apud Ferreira et al., 2018).

Estudos com familiares de pessoas com esquizofrenia expõem os impactos ocasionados na vida dos familiares, especificamente: situações de medo, estresse, cansaço, agressividade e preocupação resultando em desgaste físico e mental, bem como os prejuízos sociais e psíquicos que dificultam o convívio da família com o ser adoecido. Aponta ainda a família como elo fundamental no apoio, acolhimento e recuperação das pessoas com esquizofrenia. (Carvalho et al., 2017 apud Branco et al., 2019).

Desse modo, faz-se necessário estabelecer estratégias de cuidados de enfermagem com esses pacientes, sendo assim, traçou-se como objetivo desta pesquisa, é relatar a importância do cuidado de enfermagem frente ao paciente esquizofrênico. Através de um estudo bibliográfico de caráter descritivo. E assim beneficiar tanto os profissionais, gestores como os próprios pacientes que são os que mais saem beneficiados quando se traçam novas metas e formas de cuidar e descrevendo atividade da enfermagem frente ao tratamento de pacientes esquizofrênicos.

\section{Metodologia}

Trata-se de uma pesquisa bibliográfica de caráter descritivo. Os critérios de inclusão foram, artigos, manuais do Ministério da Saúde, monografias, dissertações, teses disponíveis na íntegra, publicados e escritos na língua portuguesa, coerentes com o tema da pesquisa e publicados no período de 2015 à 2021. Os critérios de exclusão foram: materiais que não abordavam a temática proposta e/ou que não foram publicados entre os anos de 2015 a 202, não atendiam aos critérios de inclusão descritos anteriormente e fora do período estipulado para a pesquisa.

A consulta foi realizada nas seguintes bases de dados: Literatura Latino-americano e do Caribe em Ciências da Saúde (LILACS), Scientific Eletronic Library Online (SciELO), BDENF- Bireme e Sistema de Informações da OMS (Organização Mundial da Saúde). Os descritores em Ciências da Saúde (DeCS) utilizados foram: "Enfermagem"; "Saúde Mental" e "Esquizofrenia". Como toda pesquisa envolve algum tipo de risco, a pesquisa em questão envolve dados secundários e o risco é inexistente a perda de dados. A pesquisa irá beneficiar os profissionais da saúde, especificamente os enfermeiros, comunidade acadêmica em geral. Para avaliação dos dados, será utilizado programas Microssoft office e word 2016, tanto para realização das tabelas com os artigos encontrados como para a elaboração dos textos e assim, evidenciar melhor os resultados.

Foram identificados 875 artigos, sendo encontrados 167 na base de dados SciELO, 39 na Bdenf, 669 na base de dados Lilacs. Após serem aplicados os critérios de elegibilidade e exclusão, foram selecionados 09 artigos para compor o corpo deste trabalho. 
Research, Society and Development, v. 10, n. 16, e300101623918, 2021

(CC BY 4.0) | ISSN 2525-3409 | DOI: http://dx.doi.org/10.33448/rsd-v10i16.23918

Figura 1. Fluxograma de seleção de artigos utilizados.

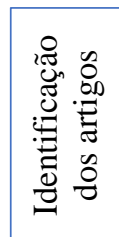

Lilacs: 669

BDENF: 39

Scielo: 167

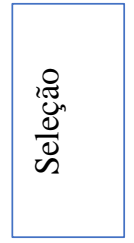

Artigos incluídos pelos
critérios de palavras
chaves: 119

Total excluídos: 839

Amostra duplicados: 01

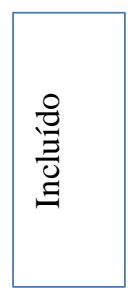

\begin{tabular}{|l|}
\hline Total de artigos \\
selecionados: 09 \\
\hline
\end{tabular}

Fonte: Autores (2021).

\section{Resultados}

A seguir, no Quadro 1 são apresentados os resultados do estudo desenvolvido. São apresentadas as principais informações coletadas identificando os artigos a partir de autores, ano de publicação, título da pesquisa, objetivos e desfechos.

Quadro 1. Representação dos artigos selecionados para o estudo, segundo os critérios de inclusão e exclusão.

\begin{tabular}{|c|c|c|c|c|}
\hline Autor & Ano & Título & Objetivo & Desfecho \\
\hline $\begin{array}{l}\text { CARDOSO; } \\
\text { CARVALHO; } \\
\text { MATOS }\end{array}$ & 2020 & $\begin{array}{l}\text { A prática de } \\
\text { enfermagem frente aos } \\
\text { pacientes portadores de } \\
\text { esquizofrenia }\end{array}$ & $\begin{array}{l}\text { Compreender a prática de enfermagem } \\
\text { frente aos pacientes portadores de } \\
\text { esquizofrenia, considerando que o } \\
\text { cuidado humanizado do enfermeiro } \\
\text { junto a esse paciente é de extrema } \\
\text { importância, pois visa a uma } \\
\text { assistência holística estabelecendo } \\
\text { vínculos do paciente e seus familiares. }\end{array}$ & $\begin{array}{l}\text { A enfermagem tem que desenvolver-se na área psiquiátrica } \\
\text { e investir em pesquisa visto que tem pouco a trabalhos } \\
\text { científicos publicados sobre o tema melhorando assim a } \\
\text { assistência prestada, pois a mesma será baseada em } \\
\text { conhecimento específico de Enfermagem. }\end{array}$ \\
\hline $\begin{array}{l}\text { FAGUNDES; } \\
\text { CAMPOS; } \\
\text { FORTES }\end{array}$ & 2021 & $\begin{array}{l}\text { Matriciamento em } \\
\text { Saúde Mental: análise } \\
\text { do cuidado às pessoas } \\
\text { em sofrimento } \\
\text { psíquico na Atenção } \\
\text { Básica }\end{array}$ & $\begin{array}{l}\text { Analisar a qualificação do cuidado em } \\
\text { SM na AB através das ações de Apoio } \\
\text { Matricial em Saúde Mental (AMSM). }\end{array}$ & $\begin{array}{l}\text { Verificou-se que as ações de matriciamento ainda estão } \\
\text { sendo implementadas de maneira insuficientes nas } \\
\text { diferentes unidades do SUS. Os resultados demonstram } \\
\text { que o cuidado básico em saúde mental se faz presente } \\
\text { somente de } 55 \% \text { a } 73 \% \text { das equipes. }\end{array}$ \\
\hline FREITAS & 2018 & $\begin{array}{l}\text { A Evolução da Saúde } \\
\text { Mental no Brasil: } \\
\text { Reinserção Social }\end{array}$ & $\begin{array}{l}\text { Abordar sobre a evolução da Saúde } \\
\text { Mental no Brasil. }\end{array}$ & $\begin{array}{l}\text { Foi possível perceber que houve grandes avanços na forma } \\
\text { como os pacientes com transtorno mentais são assistidos. }\end{array}$ \\
\hline MORAIS & 2021 & $\begin{array}{l}\text { A Esquizofrenia e o } \\
\text { Papel do Enfermeiro à } \\
\text { Adesão no Tratamento: } \\
\text { Umarararo } \\
\text { Integrativa. Revisão }\end{array}$ & $\begin{array}{l}\text { Avaliar o papel do enfermeiro frente ao } \\
\text { atendimento do usuário nos serviços de } \\
\text { saúde diagnosticado com } \\
\text { esquizofrenia. }\end{array}$ & $\begin{array}{l}\text { Nota-se que o papel do enfermeiro é fundamental no } \\
\text { cuidado ao paciente esquizofrênico, pois se direciona tanto } \\
\text { para a identificação dos sinais e sintomas quanto para a } \\
\text { avaliação das necessidades do indivíduo. }\end{array}$ \\
\hline
\end{tabular}




\begin{tabular}{|c|c|c|c|c|}
\hline $\begin{array}{l}\text { PEREIRA; } \\
\text { FRAGA; } \\
\text { NUNES }\end{array}$ & 2021 & $\begin{array}{l}\text { Percepção do paciente } \\
\text { esquizofrênico quanto } \\
\text { às modalidades de } \\
\text { atendimento nos } \\
\text { serviços de saúde } \\
\text { mental }\end{array}$ & $\begin{array}{l}\text { Conhecer a percepção do paciente } \\
\text { esquizofrênico, usuário do Centro de } \\
\text { Atenção Psicossocial II, quanto às } \\
\text { modalidades de atendimento hospitalar } \\
\text { e comunitário }\end{array}$ & $\begin{array}{l}\text { Conforme a percepção dos entrevistados, a fundamental } \\
\text { relevância da abordagem e tratamento nas duas } \\
\text { modalidades de atendimento. Eles expõem o quanto isto } \\
\text { contribui para melhor qualidade de vida, o quanto eles se } \\
\text { sentem importantes por serem ouvidos e compreendidos, } \\
\text { podendo conviver com suas famílias. }\end{array}$ \\
\hline $\begin{array}{l}\text { RIOS; } \\
\text { CARVALHO }\end{array}$ & 2021 & $\begin{array}{l}\text { Educação permanente } \\
\text { em saúde mental: } \\
\text { percepção da equipe de } \\
\text { enfermagem. }\end{array}$ & $\begin{array}{lcrr}\text { Compreender } & \text { a } & \text { percepção } & \text { dos } \\
\text { trabalhadores } & \text { da } & \text { equipe } & \text { de } \\
\text { enfermagem sobre a educação } & \text { a sormanente em saúde mental. }\end{array}$ & $\begin{array}{l}\text { A educação permanente em saúde mental permite que a } \\
\text { equipe de enfermagem repense as práticas, o processo de } \\
\text { trabalho e o cuidar em saúde mental, de modo mais } \\
\text { humanizado e em conformidade com a Reforma } \\
\text { Psiquiátrica. }\end{array}$ \\
\hline $\begin{array}{l}\text { SPAGOLLA; } \\
\text { COSTA }\end{array}$ & 2021 & $\begin{array}{l}\text { A atuação da } \\
\text { enfermagem na } \\
\text { assistência ao portador } \\
\text { de esquizofrenia no } \\
\text { ambiente familiar }\end{array}$ & $\begin{array}{l}\text { Conhecer de que forma a prestação de } \\
\text { assistência da enfermagem no } \\
\text { atendimento } \text { de } \quad \text { pessoas } \\
\text { esquizofrênicas pode contribuir na } \\
\text { relação com a família durante o } \\
\text { processo do tratamento. }\end{array}$ & $\begin{array}{l}\text { A abordagem do enfermeiro a pacientes portadores de } \\
\text { esquizofrenia e seus familiares é peça imprescindível no } \\
\text { acompanhamento e tratamento, visando melhor qualidade } \\
\text { do atendimento e tem papel fundamental como mediador. }\end{array}$ \\
\hline $\begin{array}{l}\text { TEIXEIRA et } \\
\text { al. }\end{array}$ & 2020 & $\begin{array}{lr}\text { Necessidades de Saúde } \\
\text { Mental res } \\
\text { Adolescentes e os } \\
\text { Cuidados rera } \\
\text { Enfermagem: } \\
\text { Integrativa. }\end{array}$ & 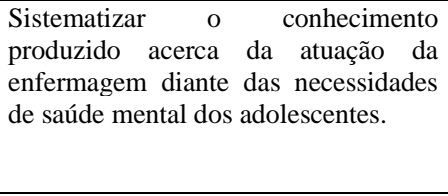 & $\begin{array}{l}\text { Permite ao profissional a formulação de novas ações que } \\
\text { se baseiem nas reais necessidades de saúde mental do } \\
\text { adolescente, que, por não frequentar tanto os serviços de } \\
\text { saúde, acaba sendo negligenciado. }\end{array}$ \\
\hline
\end{tabular}

Fonte: Autores (2021).

\section{Discussão}

\subsection{Histórico Saúde Mental no Brasil}

De modo histórico, a pessoa com doença mental necessitava ser excluso da sociedade a partir do momento em que era considerado com loucura sendo considerado um indivíduo que foge dos padrões ditos como normais. Sendo assim, não existia tratamento para a recuperação dessas pessoas não havia também a preocupação para a criação de tratamentos. (Saraiva \& Santos; Sousa, 2016 apud Freitas, 2018).

Com a chegada da família real no Brasil as pessoas que eram portadoras que qualquer distúrbio mental e eram de famílias tradicionais, eram tratadas em abrigos. Estes asilos tinham o objetivo de afastar esses indivíduos do convívio social, pois estas pessoas traziam para os seus familiares espanto e vergonha se ficasse em convívio com a família. (Santos \& Miranda, 2015 apud Freitas, 2018).

A forma de anular as pessoas que possuem doença mental se deu por muitos anos. Exclusão esta que ocasionou com o acolhimento das pessoas com transtorno mental em ambientes isolados tratando suas patologias em locais de contenção por causa de suas diferenças. Esses hospitais psiquiátricos não tinham o objetivo de prestar assistência humanizada e sim de resolver os problemas causados pelos anormais, ou seja, os manicômios eram associados com modelos prisionais e não terapêuticos. (Santos; Miranda, 2015 apud Freitas, 2018).

Finalmente, em 2001 a Lei da Psiquiátrica foi promulgada, a Lei 10.216 que mudou o modelo de assistência em mental no Brasil, com isso as pessoas com transtorno mental tiveram direitos como cidadãos. Tal lei fala dos direitos desses indivíduos, sua autonomia, da internação sem necessidade e internação voluntária e involuntária e principalmente da inclusão da pessoa no meio social. Sabendo que essa pessoa necessita de inclusão e tratamento humanizado para que assim consiga ter uma melhor qualidade de vida. (Brasil, 2001).

\subsection{Esquizofrenia}

Segundo a Organização Mundial de Saúde (1998) a esquizofrenia é conhecida como uma das doenças psiquiátricas mais desafiadoras e graves e ainda por muitos a ser estudada até hoje. Segundo a Classificação Internacional das Doenças é uma enfermidade complexa, caracterizada por distorções do pensamento, da percepção de si mesmo e da realidade externa, além de inadequação e embotamento do afeto. É uma doença que atinge cerca de $1 \%$ de população mundial, segundo a Organização 
Mundial de Saúde, ou seja, aproximadamente 70 milhões de pessoas em todo o mundo. A esquizofrenia é subdividida em categoria, na qual cada uma guarda características peculiares. Sendo essas descritas no quadro a seguir:

Quadro 2. Subtipos de esquizofrenia.

\begin{tabular}{|c|l|}
\hline TIPOS & \multicolumn{1}{c|}{ DESCRIÇãO } \\
\hline ESQUIZOFRENIA PARANÓIDE & $\begin{array}{l}\text { Definida pela presença de ideias delirantes, consequentemente de perseguição, em geral seguida de alucinações } \\
\text { e de perturbações e percepções. Seus portadores são sujeitos tensos, desconfiados, hostis e muito agressivos, } \\
\text { podendo cometer ações de violência. }\end{array}$ \\
\hline ESQUIZOFRENIA HEBEFRÊNICA & $\begin{array}{l}\text { Caracteriza-se pela presença proeminente de uma perturbação dos afetos. Ainda que, o pensamento é } \\
\text { desorganizado, o } \\
\text { discurso incoerente e há uma tendência ao isolamento social. }\end{array}$ \\
\hline ESQUIZOFRENIA CATATÔNICA & $\begin{array}{l}\text { Apresentada por distúrbios psicomotores proeminentes que podem mudar entre extremos tais como } \\
\text { hipercinesia e estupor, ou entre a obediência automática e o negativismo. }\end{array}$ \\
\hline ESQUIZOFRENIA RESIDUAL & $\begin{array}{l}\text { É definida pela presença persistente de sintomas } \\
\text { "negativos", como mudanças no comportamento, nas emoções e na interação social, embora não } \\
\text { inevitavelmente irreversíveis e não com a mesma frequência dos outros tipos de esquizofrenia. }\end{array}$ \\
\hline
\end{tabular}

Fonte: Nunes et al. (2020).

As manifestações clínicas da esquizofrenia e dos transtornos esquizofrênicos são várias e podem haver mudanças com o passar do tempo devido a sua grande diversidade. Muitos sintomas são nítidos, como alucinações, outros são relativamente sutis, como achatamento afetivo ou incongruência e podem passar despercebidos por um observador. (Yaleniz \& Velasco, 2018 apud Morais, 2021).

O transtorno esquizofrênico a prevalência é igual entre homens e mulheres; porém no homem, o problema precoce inicialmente, entre 10 e 25 anos; geralmente a primeira internação acontece antes dos 25 anos. Em mulheres a idade de maior pico da prevalência da doença é entre 25 e 30 anos, fazendo com que o prognóstico seja mais favorável para a mulher em relação ao homem, por ser de início mais tardio. A manifestação da esquizofrenia é de forma insidiosa ou aguda, contudo visa-se uma fase prodrômica indicada por isolamento social e vivências com ausência de respostas emocionais. (Carvalho et al., 2017).

Segundo (Spagolla \& Costa, 2020) observaram que a esquizofrenia afeta quase todos os aspectos das funções psíquicas, tratando-se de uma doença crônica, de causas praticamente desconhecidas, representando grandes desafios para o paciente, família e o sistema de saúde. São muitas as dificuldades dos familiares que convivem com os esquizofrênicos para entender o distúrbio. $\mathrm{O}$ acolhimento essas famílias fornecendo informações sobre a doença estabelece impactos positivos gerando melhora para o paciente e a sua família.

Essa patologia abrange de $1 \%$ da população mundial e frequentemente inicia-se entre o fim da adolescência e por volta dos 30 anos. O início pode ser gradual ou agudo, apesar da maioria das pessoas possuem um desenvolvimento lento e manifeste muitos sinais e sintomas, pode ser ou não antecedido de um evento estressante (Assunção, 2016 apud Morais, 2021).

Os Centros de Atenção Psicossocial constituem-se como serviços comunitários e ambulatoriais regionalizados, nos quais os usuários devem receber cuidados médicos, atendimentos terapêuticos individuais e/ou grupais em que a inclusão dos familiares é uma iniciativa fundamental e as questões de ordem sociais presente no cotidiano dos usuários. (Pereira et al., 2021).

\subsection{Matriciamento}

O matriciamento ou apoio matricial compreende a construção compartilhada de uma proposta interventiva pedagógicoterapêutica, envolvendo duas ou mais equipes, que se reorganizam em equipe de referência e equipe de apoio matricial. Objetivase promover uma retaguarda especializada à assistência, ao vínculo interprofissional e ao apoio institucional no desenvolvimento coletivo de projeto terapêutico junto a indivíduos, famílias e comunidade. (Chiaverini et al., 2017 apud Fagundes; Campos; Fortes, 2021). 
O matriciamento em saúde mental simboliza prática interdisciplinaridade com fabricação da saúde no território que privilegia a articulação entre as Unidades Básicas de Saúde da Família (UBSF), responsáveis pela ordenação dos fluxos assistenciais no sistema de saúde, e os Centros de Atenção Psicossocial (CAPS), dispositivos substitutivos prioritários na composição da RAPS. (Chiaverini et al., 2017 apud Fagundes et al., 2021).

\subsection{Importância da Atenção de Enfermagem}

Ao constatar as necessidades de saúde mental dos indivíduos, é possível traçar potenciais diagnósticos que financiem a elaboração da sistematização de enfermagem, analisando-se os fatores de risco, sinais, sintomas, e possíveis causas que provocam agravos. Para elaboração dos diagnósticos, é preciso encontrar aspectos importantes das necessidades de saúde dos pacientes, sendo esses dados colhidos a partir da anamnese e de exames físicos e mentais. (Teixeira et al., 2020).

É necessário dar uma atenção holística aos clientes com transtornos mentais desde a entrada até sua alta hospitalar, conduzindo sua evolução rotineiramente através do monitoramento dos sinais e sintomas. É de grande importância que os profissionais de enfermagem tenham prática e conhecimento técnico científico na identificação da Esquizofrenia em pacientes. hospitalizados, por ser uma assistência de enfermagem sistematizada, tendo em vista um cuidado qualificado a estes clientes, executando as intervenções aceitáveis para que eles tenham uma melhor qualidade de vida. (Cardoso et al., 2020)

A comunicação evolui no enfermeiro a capacidade de aproximar-se das pessoas ao seu redor, quando exercício de suas atividades profissionais. Por meio do uso de modelos de enfermagem e teorias para o paciente de planejamento e de saúde cuidados, os enfermeiros serão capazes de ofertar um serviço melhor para indivíduo e da comunidade (Santos, 2017).

No entanto, há estudos que mostram que os sentimentos de empatia, respeito e paciência são essenciais e primordiais entre o paciente com esquizofrenia e o cuidador, enfatizando que a comunicação como eixo fundamental no fortalecimento do relacionamento construído ao longo do tempo, destacando a família como peça no tratamento da esquizofrenia (Assunção et al., 2016 apud Morais et al., 2021).

Ainda há a falta de capacitação continuada na área de saúde mental, para que os profissionais se sintam habilitados para atender estes casos, pois estes profissionais não tinham hábitos de adquirir conhecimento específicos sobre saúde mental. Com isso, há a necessidade da criação de estratégias para o enfrentamento das necessidades desta população. (Rios; Carvalho, 2021).

\section{Considerações Finais}

Através desta pesquisa foi possível conhecer sobre a evolução dos tratamentos psiquiátricos e um pouco mais sobre transtornos esquizofrênicos. Sobretudo, este trabalho possibilitou o alcance de uma visão holística sobre a atuação de enfermagem frente aos cuidados com esses pacientes, não apenas com enfoque técnico-medicamentoso. Ao que se pode perceber, a SAE é favorável para um bom prognóstico. Além do mais nota-se, que a melhor assistência é a individualizada e humanizada, assistindo o paciente como um ser único, respeitando as suas necessidades biopsicossociais.

Pode-se entender que a promoção do cuidado de enfermagem não absolutamente é feita apenas por meio de artifícios técnicos, em um leito hospitalar ou em um ambulatório, considerando-se, após a reforma psiquiátrica ter possibilitado ao paciente esquizofrênico a convivência familiar e em meio social. O respeito, a escuta atenta, à vontade de interagir, o vínculo, a confiança são elementos que precisam ser usados na prestação de assistência qualificada, especialmente em pacientes psíquicos.

Existe um sentido contrário à esquizofrenia em relação a outras doenças por ser pouco divulgada e cercada de muitos mitos e estigmas, que acaba ocasionando ainda mais sofrimento para o portador e sua família que precisam aprender a lidar com o impacto causado por essa patologia. 
Portanto, é necessário que seja trabalhado a implantação da sistematização de enfermagem em ambiente que atendem pacientes com esquizofrenia e ainda não é trabalhado este processo, para que haja desenvolvimento terapêutico desses pacientes pois o enfermeiro é capaz de identificar os anseios do paciente, elaborar planos de cuidado e ainda observar se esses planos demonstram os resultados esperados.

O enfermeiro tem uma importância considerável em direcionar os cuidados com o paciente afetado pela esquizofrenia, ainda que exista uma dificuldade própria do transtorno, seus cuidados favoreciam não somente o paciente em tratamento, mas toda uma coletividade que faz parte da vida desse indivíduo.

\section{Referências}

Amarante, P., \& Nunes, M. D. O. (2018). A reforma psiquiátrica no SUS e a luta por uma sociedade sem manicômios. https://www.scielosp.org/article/csc/2018.v23n6/2067-2074/pt/

Branco, F. M. F. C., da Silva, J. B., Dutok, C. M. S., \& Neto, T. C. B. (2019). Percepção dos familiares de pessoas com esquizofrenia acerca da doença. Revista Eletrônica Acervo Saúde, 11(12), e944-e944. https://acervomais.com.br/index.php/saude/article/view/944

Brasil. (2001) Ministério da Saúde. Lei n. ${ }^{\circ}$ 10216, de 06 de abril de 2001. Dispõe sobre a proteção e os direitos das pessoas portadoras de transtornos mentais e redireciona o modelo assistencial em saúde mental. Brasília: MS.

Brasil. (2014) Ministério da Saúde. Núcleo de Apoio à Saúde da Família Volume 1: ferramentas para a gestão e para o trabalho cotidiano. Cadernos de Atenção Básica (39). Brasília: MS. https://bvsms.saude.gov.br/bvs/publicacoes/nucleo_apoio_saude_familia_cab39.pdf

Cardoso, A. O. J., Carvalho, G. T., \& Matos, T. S. (2020) A prática de enfermagem frente aos pacientes portadores de esquizofrenia. Revista Eletrônica Acervo Enfermagem 5, e5118-e5118

Carvalho, C. M. S., Sousa, D. M. G., Pinho, R. I. A., Fernandes, M. A., \& Oliveira, A. D. S. (2017). Vivências de familiares da pessoa com esquizofrenia. SMAD Revista Eletrônica Saúde Mental Álcool e Drogas (Edição em Português), 13(3), 125-131. https://www.revistas.usp.br/smad/article/view/149377

Fagundes, G. S., Campos, M. R., \& Fortes, S. L. C. L. (2021). Matriciamento em Saúde Mental: análise do cuidado às pessoas em sofrimento psíquico na Atenção Básica. Ciência \& Saúde Coletiva, 26, 2311-2322. https://www.scielo.br/j/csc/a/McmFdYbq6pRgTMqJXtzVfbP/?lang=pt\#

Ferreira, J. T. M. A., Laranjeira, A., Mata, M. A., \& Pimentel, M. H. (2018). Problemas dos cuidadores de doentes com esquizofrenia: a sobrecarga familiar. Revista Portuguesa de Enfermagem de Saúde Mental, 19, 8-16. https://bibliotecadigital.ipb.pt/handle/10198/18064

Freitas, L. B. (2018). A evolução da saúde mental no brasil: reinserção social. Revista Científica Semana Acadêmica, 126(1). https://semanaacademica.org.br/artigo/evolucao-da-saude-mental-no-brasil-reinsercao-social

Macedo, M., Marques, A., Queirós, C., \& Mariotti, M. C. (2018). Esquizofrenia, atividades instrumentais de vida diária e funções executivas: uma abordagem qualitativa1. Cadernos Brasileiros de Terapia Ocupacional, 26, 287-298. https://www.scielo.br/j/cadbto/a/XSz4YNCy3tkWcs6L4jq46jD/?lang=pt

Morais, A. L. J. Teles, W. S. Silva, M. C. Torres, R. C. Barros, A. M. M. S. Andrade, A. F. S.M. Azevedo, M. V. C. Debbo, A. Junior, P. C. C. S. Calasans, T. A. S. Silva, \& M. H. S. (2021). A esquizofrenia e o papel do enfermeiro à adesão no tratamento: Uma revisão integrativa. Research, Society and Development, 10(9), e43810918305-e43810918305. https://rsdjournal.org/index.php/rsd/article/view/18305

Organização Mundial da Saúde. (1998). CID-10: classificação de transtornos mentais e de comportamento a CID-10: critérios diagnósticos para pesquisa. Porto Alegre: Artes Médicas, 82- 3.

Pereira, V. P., Fraga, V. S., \& Nunes, G. (2021). Percepção do paciente esquizofrênico quanto às modalidades de atendimento nos serviços de saúde mental. Global Academic Nursing Journal, 2(2), e138-e138. https://globalacademicnursing.com/index.php/globacadnurs/article/view/248

Rios, A. D. S., \& Carvalho, L. C. D. (2021). Educação permanente em saúde mental: percepção da equipe de enfermagem. Rev. enferm. UFPE on line, 1-23. https://pesquisa.bvsalud.org/portal/resource/pt/biblio-1145786.

Rosa, D. C. J., Lima, D. M. D., Miranda, L., \& Peres, R. S. (2021). “Paciente-problema”: imaginário coletivo de enfermeiros acerca do usuário com diagnóstico de esquizofrenia. Physis: Revista de Saúde Coletiva, 31. https://www.scielo.br/j/physis/a/nm8VStnPRNYcvZBVLZfsd6H/

Santos, A. D. (2017). Papel do Enfermeiro frente ao cuidado de pacientes esquizofrênicos. https://repositorio.faema.edu.br/handle/123456789/2092

Spagolla, K. C., \& Costa, M. O. (2021). A atuação da enfermagem na assistência ao portador de esquizofrenia no ambiente familiar. Research, Society and Development, 10(7), e30410716601-e30410716601. https://rsdjournal.org/index.php/rsd/article/view/16601

Teixeira, L. A., Freitas, R. J. M. D., Moura, N. A. D., \& Monteiro, A. R. M. (2020). Necessidades de saúde mental de adolescentes e os cuidados de enfermagem: revisão integrativa. Texto \& Contexto-Enfermagem, 29. https://www.scielo.br/j/tce/a/sxfq53q5mHTcVrXRmmXdKSp/abstract/?lang=pt 